\title{
Adicción al móvil e internet en adolescentes y su relación con problemas psicopatológicos y variables protectoras
}

\section{Mobile and internet addiction in adolescents and its relationship with psychopathological problems and protective variables}

\author{
José Luis Vicente-Escudero, Pedro Saura-Garre, Concepción López-Soler, Antonia Martínez y Mavi \\ Alcántara \\ Universidad de Murcia
}

\begin{abstract}
Resumen
Se analizó la relación entre psicopatología y abuso del móvil e internet en adolescentes, además, se analizaron si ciertas variables protectoras de psicopatología protegen también del abuso del móvil e internet y se calcularon los puntos de corte dos cuestionarios que miden el abuso de estas tecnologías. Se empleó una muestra de 269 adolescentes entre 12 y 18 años (124 chicos y 145 chicas) y se usaron los cuestionarios YSR para medir psicopatología, CERI abuso de internet y CERM abuso del móvil. Los resultados mostraron que el abuso de las nuevas tecnologías se relaciona con sintomatología externalizante y de déficit de atención. Se halló que el número de clubs a los que pertenecen, relación con los padres, autonomía y rendimiento escolar en ciencias, son variables protectoras frente al abuso del móvil e internet. Además, a los 15 años aumenta significativamente el uso de estas tecnologías y la psicopatología asociada.
\end{abstract}

Palabras Clave: Psicopatología, móvil, internet, adolescentes, protección.

\begin{abstract}
The objectives of this study were to analyse the relationship between psychopathological problems and mobile phone and internet abuse in adolescents, and whether certain variables that offer protection from psychopathological problems also offer protection against this type of abuse. We administered two questionnaires that measure such abuse. The study included a sample of 269 adolescents aged between 12 and 18 years (124 boys and 145 girls). The YSR, CERI, and CERM questionnaires were used to measure psychopathology, internet abuse, and mobile abuse, respectively. The results showed that the abuse of new technologies is particularly related to externalizing symptoms and attention deficit. It was found that variables such as the number of clubs to which the participants belonged, their relationship with parents, autonomy, and school performance in science are protective variables against mobile phone and internet abuse. At the age of 15 years, there was a significant increase in the use of these technologies and their associated psychopathological problems.
\end{abstract}

Keywords: psychopathology, mobile, internet, adolescents, protection.

Cómo citar este artículo: Vicente-Escudero, J. L., Saura-Garre, P.; López-Soler, C., Martínez, A., y Alcántara, M. (2019). Adicción al móvil e internet en adolescentes y su relación con problemas psicopatológicos y variables protectoras. Escritos de Psicología, 12, 103-112.

Correspondencia: José Luis Vicente Escudero. Hospital Universitario Virgen de Arrixaca. El Palmar. Murcia, España. E-mail: joseluis.vicente@um.es. E-mail de la coautora Concepción López Soler: clopez@um.es. 


\section{Introducción}

Estamos ante una nueva era digital, determinada por nuestra capacidad de expresarnos en ceros y unos (Roca, 2015) en la que las tecnologías como internet o el teléfono móvil se han convertido en un eje central en la vida de muchas personas por diferentes razones: ya están en casi todos los hogares, facilitan el trabajo, el acceso a la información, el conocimiento del entorno, exigiendo mucha dedicación y atención (Labrador y Villadangos, 2009).

Numerosos autores se han aventurado a realizar su definición de nuevas tecnologías, que algunos caracterizan como "las herramientas diseñadas para informar y comunicar, de una manera sencilla y que tienen como característica principal una accesibilidad permanente" (Beranuy et al., 2009; Castellana et al., 2007).

Poder acceder a toda la información que se desee independientemente de dónde se haya producido es una de las características más favorables de las nuevas tecnologías. Sin embargo, las nuevas tecnologías albergan en sí mismas aspectos negativos, existiendo un generalizado criterio entre profesionales de la salud sobre el potencial de dichas tecnologías para incurrir en un uso inadecuado de las mismas, sobre todo por población vulnerable, y en casos concretos, para generar una auténtica adicción (Instituto de Adicciones de Madrid Salud, 2008).

En términos generales, se puede decir que la adolescencia es un colectivo sumamente susceptible de sufrir patrones de conductas adictivas u otros trastornos psicológicos, como uso abusivo de las nuevas tecnologías, problemas de conducta o abuso de drogas (Castellana et al., 2007).

Los datos del último año del Instituto Nacional de Estadística (INE, 2019) muestran que la proporción de menores que usan las nuevas tecnologías es muy elevada. En dicho informe se observa que el uso de ordenadores e internet es algo prácticamente universal para el colectivo de los adolescentes. Atendiendo más concretamente al uso que éstos hacen entre los 12 y 16 años, se observa un discreto aumento del uso del internet conforme va aumentando la edad, comenzando a los 12 años con un uso de internet del $92.4 \%$ y un $98 \%$ a los 15 años. Se encuentran diferencias más pronunciadas en cuanto a tener un teléfono móvil en propiedad, a los 12 años el $63.9 \%$ de los adolescentes dispone de un móvil, a los 13 años ya dispone de móvil el $84 \%$, hasta llegar a los 15 años cuando tienen un teléfono inteligente el $93.8 \%$ de los adolescentes. Al llegar al rango de edad entre los 16 y 24 años, prácticamente el total de la población usa diariamente internet (99.1\%) y el móvil (99.4\%).

Además, según algunos autores (Echeburúa et al., 2009; Labrador y Villadangos, 2009) el tiempo total diario que los adolescentes podrían hacer del uso de los aparatos electrónicos como el móvil, el ordenador o la televisión, podría superar las 6 horas diarias. Un uso que podría incrementarse más durante los fines de semana y días festivos.

El móvil favorece la autonomía personal, proporciona identidad y prestigio y es una fuente de ocio (Chóliz y Villanueva, 2011), estos motivos podrían explicar este incremento tan notable en su uso y hacerlo tan atractivo.

A pesar de la gran cantidad de tiempo que los adolescentes dedican a las nuevas tecnologías, existen estudios que muestran ciertas características positivas que pueden derivarse de este uso. Por ejemplo, el uso del teléfono móvil se relaciona con una mejor socialización al comportarse éste como un facilitador de las relaciones interpersonales, permite una subjetiva sensación de proximidad y promueve el sentimiento de pertenencia al grupo; pudiendo ser usado como un mecanismo de diferenciación social (Cuesta y Gaspar, 2014). Otros estudios (Oliva et al., 2012) apuntan que las redes sociales tienen algunas consecuencias positivas en la creación y mantenimiento de las relaciones sociales.

Entre las principales consecuencias negativas de un uso inadecuado, Espinar y López, (2009) destacan algunos riesgos importantes en estos rangos de edad: el acceso a contenidos inapropiados, contacto con desconocidos, amenazas a la privacidad así como ciertos peligros relacionados con el comercio electrónico. Hay otros autores que enfatizan que con un excesivo uso de las nuevas tecnologías existe un alto riesgo de generar un comportamiento adictivo que curse no ya con una dedicación desmedida a las mismas, sino con una verdadera dependencia y falta de control sobre estas conductas (Gil et al., 2003; Oliva et al., 2012; Young, 2004).

Son numerosas las investigaciones que han analizado las consecuencias del uso problemático de las nuevas tecnologías en la población española. En cuanto a las prevalencias del uso problemático de internet y del móvil, llama la atención la variabilidad existente entre los estudios, oscilando desde el $2 \%$ de usuarios problemáticos (Rial et al., 2014), hasta el 19,9\% hallado por Gómez et al. (2014) para el caso de internet, mientras que para el móvil oscilan entre el 6,1\% encontrado en el estudio de Carbonell, et al. (2012) hasta el 14,8\% que encuentran López-Fernández et al. (2012). Esta variabilidad 
podría ser debida a las diferencias metodológicas entre estudios, tipo de muestra o variedad de instrumentos de medida empleados.

En cuanto a las consecuencias que el uso inadecuado puede ocasionar en los menores, se ha encontrado que aquellos adolescentes que abusan de internet presentan mayores problemas de ansiedad, insomnio y depresión (Estévez et al., 2009; Jenaro et al., 2007; Wu et al., 2019), comportamiento impulsivo (Cerniglia et al., (2019), mayor malestar psicológico (Fumero et al., 2020), con recurrentes pensamientos negativos que pueden interferir en situaciones sociales, así como relación con ideación suicida (Arrivillaga et al., 2020).

El género no parece estar relacionado con el uso problemático de internet aunque los varones tengan un uso más alto de las nuevas tecnologías (Estévez et al., 2009; Muñoz-Rivas et al., 2003; Viñas et al., 2002; y Rial et al., 2014). El autocontrol parece ser una de las variables que tiene una relación más alta con el abuso de las nuevas tecnologías (Oliva et al., 2012): en los últimos años algunos autores han mostrado que la relación entre TDAH y adicción a las nuevas tecnologías es moderadamente alta (Andreassen et al., 2017) debido a las características del trastorno de déficit de atención e hiperactividad tales como el deficiente aprendizaje del autocontrol y las dificultades para retardar la gratificación y tolerar la frustración (López-Soler y Romero, 2013). En los últimos años algunos autores han mostrado que la relación entre TDAH y adicción a las nuevas tecnologías es moderadamente alta (Andreassen et al., 2017).

Además, autores como Beranuy et al. (2009), Carbonell et al. (2009), Chóliz et al. (2009), Jenaro, et al. (2007), Labrador y Villadangos, (2010) y Sánchez-Martínez y Otero (2009), encuentran que un uso intensivo del teléfono móvil está relacionado con el posterior consumo de drogas, fracaso escolar, ansiedad e insomnio, siendo las mujeres las que suelen presentar mayores dificultades.

Teniendo en cuenta lo anterior, los objetivos que el presente estudio plantea son los siguientes:

a) Conocer la magnitud de la relación existente entre problemas psicopatológicos y el uso que hacen los adolescentes de las nuevas tecnologías, en concreto del uso de internet y del teléfono móvil.

b) Obtener las prevalencias del uso problemático de internet y del teléfono móvil y su relación con la psicopatología en esta población, y analizar las diferencias existentes entre variables protectoras de problemas psicopatológicos, las competencias psicosociales en función del uso de internet y del teléfono móvil.

\section{Método}

\section{Participantes}

Para alcanzar los objetivos de este estudio se seleccionó una muestra de 269 estudiantes de la E.S.O, pertenecientes a población general de la comarca del Mar Menor (Región de Murcia), de los cuales 124 eran chicos (46.1\%) y 145 eran chicas (53.9\%), con edades comprendidas entre 12 y 18 años (tabla1).

Se optó por un muestreo por conglomerados, a través del curso académico en el que se encuentra escolarizada la población estudiada, lo que tuvo como consecuencia obtener alguna entrevista de algún menor de edad superior a los 16 años por la posibilidad de repetir curso.

Todos los sujetos que componían la muestra asistían a dos institutos públicos seleccionados de manera incidental en el transcurso del curso académico, 222 jóvenes del instituto "A" $(82.2 \%)$ y 48 adolescentes del instituto " $B$ " (17.8\%), fueron seleccionados por muestreo incidental.

La media de edad de los sujetos fue 14.19 años con una desviación típica (DT) de 1.49, siendo el grupo más numeroso el de 14 años ( $23 \%$ de la muestra), la moda fue ser chica para la variable sexo y el $79 \%$ de la muestra fue población nacionalizada española y el $21 \%$ población extranjera.

Tabla 1

Distribución de la muestra por edad y sexo.

\begin{tabular}{|c|c|c|c|c|c|c|}
\hline \multirow[b]{2}{*}{ Edad } & \multicolumn{2}{|c|}{ CHICOS } & \multicolumn{2}{|c|}{ CHICAS } & \multicolumn{2}{|c|}{ TOTAL } \\
\hline & $\mathrm{N}$ & $\%$ & $\mathrm{~N}$ & $\%$ & $\mathrm{~N}$ & $\%$ \\
\hline 12 & 22 & 17.7 & 18 & 12.4 & 40 & 14.9 \\
\hline 13 & 26 & 21.0 & 32 & 22.1 & 58 & 21.6 \\
\hline 14 & 26 & 21.0 & 36 & 24.8 & 62 & 23.0 \\
\hline 15 & 24 & 19.4 & 26 & 17.9 & 50 & 18.6 \\
\hline 16 & 21 & 16.9 & 23 & 15.9 & 44 & 16.4 \\
\hline 17 & 4 & 3.2 & 7 & 4.8 & 11 & 4.1 \\
\hline 18 & 1 & 0.8 & 3 & 2.1 & 4 & 1.5 \\
\hline Total & 124 & 46.1 & 145 & 53.9 & 269 & 100 \\
\hline
\end{tabular}




\section{Instrumentos}

Se han empleado tres instrumentos de medida para la realización de este estudio.

El cuestionario Youth Self-Report (YSR) para jóvenes y adolescentes entre 11 y 18 años (Achenbachy Edelbrock, 1987), en la adaptación española de Lemos, et al. (1992), consta de dos partes.

La primera parte del YSR es una encuesta sociodemográfica que busca información sobre sexo, edad, curso escolar, trabajo de los padres... Y también consta de 7 ítems en una escala de valoración que buscan información sobre competencias psicosociales como el número de amigos (ninguno, uno, dos o tres y cuatro o más) y salidas que con ellos realizan (menos de una, una o dos y tres o más), colaboración con organizaciones (ninguna colaboración, una, dos y tres colaboraciones), relación con otros jóvenes (peor, igual o mejor que los demás), con los padres (peor, igual o mejor que los demás) y con los hermanos (peor, igual o mejor que los demás), autonomía personal (peor, igual o mejor que los demás) y rendimiento en asignaturas como letras y matemáticas (suspenso, peor que el promedio, en el promedio y mejor que el promedio). En su adaptación al español, esta parte del YSR mostró una buena fiabilidad $(\alpha=.62)$.

La segunda parte del cuestionario está constituida por 112 ítems en una escala tipo Likert de 3 opciones, donde "0" es "no es cierto", " 1 " es "algunas veces es cierto" y "2" es "cierto muy a menudo". Esta parte evalúa problemas emocionales o comportamentales, tanto de banda ancha (problemas externalizantes e internalizantes) como de banda estrecha (Ansiedad/Depresión, Retraimiento/Depresión, Quejas Somáticas, Problemas Sociales, Problemas de Pensamiento, Problemas de Atención, Conducta Disocial y Conducta Agresiva), así como competencias prosociales. En su adaptación al español, esta parte del YSR mostró una alta fiabilidad $(\alpha=.90)$.

Cuestionario de Experiencias Relacionadas con Internet (CERI, Beranuy et al., 2009), pretende detectar las consecuencias intrapersonales e interpersonales del uso de internet en las personas. Consta de diez ítems en una escala tipo Likert de cuatro opciones de respuesta en orden de intensidad creciente (1= Casi nunca, 2= Algunas veces, $3=$ Bastantes veces, 4= Casi siempre). Compuesto por dos factores: conflictos intrapersonales, con 6 ítems que buscan información sobre si la persona piensa que la vida sin internet es aburrida, vacía y triste o si la persona se enfada si alguien le molesta mientras está conectado. Y el factor conflictos interpersonales, de 4 ítems que buscan información sobre si la persona cree que su rendimiento laboral o escolar se ha visto afectado por el uso que hace de la red o con qué frecuencia la persona abandona las cosas que está haciendo para estar más tiempo conectado a la red. Una mayor puntuación en los ítems indica un mayor nivel de conflicto. Este instrumento en su versión original mostró una buena fiabilidad $(\alpha=.77)$.

Cuestionario de Experiencias Relacionadas con el Móvil (CERM, Beranuyet al., 2009), es una adaptación del cuestionario CERI centrado en el abuso del móvil. Consta de diez ítems con cuatro opciones de respuesta con el mismo criterio que el cuestionario anterior. Consta también de dos factores, el factor conflictos, con 5 ítems que buscan información sobre si la persona ha tenido riesgo de perder una relación importante, trabajo u oportunidad académica por culpa del móvil o si la persona siente necesidad de invertir cada vez más tiempo en el móvil para sentirse satisfecho. Y el factor uso comunicacional y emocional, con 5 ítems que buscan información sobre hasta qué punto la persona se siente inquieta si no recibe mensajes o llamadas o si cuando la persona se aburre utiliza el móvil como una forma de distracción. Una mayor puntuación en el cuestionario indica mayor nivel de conflictos y problemas comunicacionales y emocionales. Este instrumento en su versión original mostró una buena fiabilidad $(\alpha=.80)$

Ambas escalas son una adaptación del cuestionario PRI de Gracia et al. (2002).El cuestionario CERI es básicamente igual que el PRI, pero modificando la sintaxis de algunos ítems y el cuestionario CERM es una adaptación del cuestionario CERI con ítems adaptados para el uso del móvil. El cuestionario PRI consta de 19 ítems basados en los criterios DSM-IV para el abuso de sustancias y juego patológico, incluyendo preguntas sobre aumento de tolerancia, efectos negativos, reducción de actividades, evasión, pérdida de control y deseo de ser conectado.

\section{Procedimiento}

A los participantes se les aplicó el cuestionario grupalmente, estando el investigador presente y respetando el horario de exámenes y excursiones de las clases. Participaron en el estudio aquellos adolescentes que entregaron un consentimiento informado personal y firmado por sus padres o tutores legales, en un plazo de 15 días después de que les fuera entregado el consentimiento. 
Se informó tanto a los adolescentes como al director del centro y tutores de la participación voluntaria, confidencialidad y anonimato de los datos.

El tiempo que se invirtió aproximadamente por clase en la aplicación de los cuestionarios fue de media hora aproximadamente.

\section{Análisis de Datos}

Para el tratamiento de los datos estadísticos se empleó el programa IBM SPSS versión 19 para Windows.

Se calcularon los puntos de corte para los cuestionarios CERI y CERM.

Se ha empleado el coeficiente de fiabilidad $\alpha$ de Cronbach con el fin de determinar la consistencia interna de los distintos cuestionarios utilizados.

Se han calculado las prevalencias de las alteraciones psicológicas y el uso de móvil e internet tomando como criterio Media $(M)+1 D T y M+2 D T$, en el grupo de chicas y de chicos para distintas edades.

Antes de realizar análisis univariados de varianza (ANOVAs) y estudios de relación entre variables, se ha utilizado la prueba de Kolmogorov-Smirnov para conocer si las varíables a estudiar cumplían con el principio de normalidad, y del Test de Levene para ver si se cumplía el principio de homocedasticidad.

Finalmente para poder establecer relaciones entre variables se han realizado correlaciones bivariadas a través del coeficiente rho de Spearman $(\rho)$, pues algunas variables cuantitativas no seguían la distribución normal.

\section{Resultados}

Se calcularon los puntos de corte de los cuestionarios CERI y CERM (tabla 2), siguiendo el criterio de $M+1 D T$ (posible indicador de problemática ocasional) y $M+2 D T$ (posible indicador de problemática frecuente), además, la prueba robusta de igualdad de medias para CERI $\left(X^{2}=118.05 ; p<.001\right)$ y CERM $\left(X^{2}=85.31 ; p<.001\right)$ resultó ser significativa y se encontraron diferencias significativas post hoc entre los tres grupos calculadas para cada cuestionario. La fiabilidad de los tres cuestionarios resultó ser muy buena, tanto para el YSR $(\alpha=.937)$, como para CERI $(\alpha=.830)$ y CERM $(\alpha=.820)$.

Tabla 2

Puntos de corte CERI y CERM

\begin{tabular}{lccc}
\hline & $M(D T)$ & $M+1 D T$ & $M+2 D T$ \\
\hline CERI (internet) & $18,33(5.67)$ & 24 & 29,67 \\
CERM (móvil) & $16,25(4.98)$ & 21,23 & 26,21 \\
\hline
\end{tabular}

Al analizar las prevalencias entre chicos y chicas para el uso de internet, móvil y problemas psicopatológicos, los análisis se realizaron en relación a dos grupos de edad, un grupo de 12 a 14 años y otro grupo de 15 a 18 años, debido a que tras realizar una comparación de medias tanto para el uso de internet $(F=4.43 ; p<.001)$ como para el uso del móvil $(F=3.16 ; p<.01)$ en función de la edad, el análisis post hoc mediante la prueba HDS de Tukey mostró que no existían diferencias entre las edades de 12 (CERI, M: 13.77; CERM: M: 13.33), 13 (CERI, M: 16.43; CERM: M: 14.39) y 14 años (CERI, M: 17.81; CERM: M: 16.26), pero sí que existían diferencias con el grupo de edad de 15 (CERI, M: 19.70; CERM: $M: 18.10)$ y 16 años (CERI, $M: 21.36$; CERM: $M: 17.74$ ), donde este último grupo superaba significativamente en media al grupo de edades más tempranas.

Podemos observar en las prevalencias (tabla 3 ) que los chicos aumentan con la edad la problemática frecuente relacionada con el uso de móvil e internet y disminuyen la problemática ocasional en el uso de móvil, además, tanto en la sintomatología clínica como sub-clínica hay síndromes que aumentan su presencia con la edad (como puede ser la Ansiedad-Depresión sub-clínica, el Retraimiento-Depresión clínico o la Agresividad clínica), y otros síndromes que la disminuyen(como es el caso de la Conducta Disocial clínica y la Agresividad sub-clínica)

En cambio, para las chicas se observa que aumentan con la edad la frecuencia tanto de la problemática ocasional y frecuente relacionada con el uso de móvil e internet, así como de la sintomatología clínica y sub-clínica, donde observa un incremento de prevalencias de todos los síndromes a partir de los 15-16 años. 
Tabla 3

Prevalencias por sexo y edad

\begin{tabular}{lcccccccc}
\hline & \multicolumn{3}{c}{ Chicos } & \multicolumn{2}{c}{ Chicas } \\
\hline & \multicolumn{2}{c}{ 12-14 años } & \multicolumn{2}{c}{ 15-18 años } & \multicolumn{2}{c}{ 12-14 años } & \multicolumn{2}{c}{ 15-18 años } \\
\hline Ansiedad/Depresión & 1 D.T & 2 D.T & 1 D.T & 2 D.T & 1 D.T & 2 D.T & 1 D.T & 2 D.T \\
Retraimiento/Depresión & 4.1 & 10.8 & 10 & 10 & 7 & 8.1 & 25.4 & 15.3 \\
Quejas Somáticas & 13.5 & 5.4 & 6 & 10 & 3.5 & 3.5 & 10.5 & 10.5 \\
Problemas Sociales & 4.1 & 8.1 & 6 & 10 & 9.3 & 3.5 & 21.1 & 10.2 \\
Problemas de Pensamiento & 6.8 & 5.4 & 8.3 & 4.2 & 6 & 6 & 10.7 & 12.7 \\
Problemas Atención & 2.7 & 4.1 & 8 & 2 & 3.5 & 2.3 & 15.3 & 6.8 \\
ConductaDisocial & 10.8 & 6.8 & 14 & 10 & 7 & 3.5 & 18.6 & 18.6 \\
Conducta Agresiva & 5.4 & 6.8 & 10 & 4 & 3.5 & 2.3 & 10.2 & 11.9 \\
Internet & 10.8 & 4.1 & 4 & 8 & 2.3 & 1.2 & 5.1 & 8.5 \\
Móvil & 8.3 & 2.1 & 18 & 6 & 4.4 & 3.5 & 15.5 & 12.1 \\
\hline
\end{tabular}

1 DT y 2 DT corresponden a sintomatología sub-clínica (problemática ocasional) y sintomatología clínica (problemática frecuente), respectivamente.

Antes de poder realizar algún análisis de tipo correlacional, se consideró necesario conocer si las puntuaciones de las distintas variables a estudiar seguían el patrón propio de la distribución normal, con el fin de saber cuál es el estadístico más adecuado para conocer la relación entre las variables. Y puesto que la prueba Kolmogorov-Smirnov resultó ser significativa en las variables uso de internet $(Z=2.13 ; p<.001)$, uso de móvil $(Z=2.63 ; p<.001)$, ansiedad/depresión $(Z=3.01 ; p<.001)$, retraimiento/depresión $(Z=2.37 ; p<.001)$, quejas somáticas $(Z=2.47 ; p<.001)$, problemas sociales $(Z=2.64$; $p<.001)$, problemas de pensamiento $(Z=3.23 ; p<.001)$, problemas de atención $(Z=1.53 ; p<.01)$, conducta disocial $(Z=2.70 ; p<.001)$, conducta agresiva $(Z=2.13 ; p<.001)$, síndromes externalizantes $(Z=$ $1.19 ; p<.001)$, síndromes internalizantes $(Z=2.58 ; p<.001)$ y problemas totales $Y S R(Z=1.21 ; p<.05)$, no se puede asumir normalidad en la distribución de las puntuaciones, por tanto se decidió utilizar el estadístico rho de Spearman $(\rho)$.

Atendiendo a la relación entre las puntuaciones totales de las pruebas que miden el uso de Internet y Móvil con las escalas problemáticas del YSR (tabla 4), todas ellas presentan correlaciones significativas con $p<.01$ con puntuaciones por el encima de $\rho=.190$ y máximo de $\rho=.423$. Entre las variables que existe mayor correlación, está la conducta disocial $(\rho=.374$ con internet y $\rho=.372$ con móvil), los problemas de atención ( $\rho=.360$ con internet, $\rho=.364$ con móvil), conducta agresiva $(\rho=$ .354 con internet y $\rho=.368$ con móvil) y síndromes externalizantes $(\rho=.413$ con internet y $\rho=.423$ con móvil). Se observa que las mejores correlaciones se dan con los problemas de tipo externalizante (como conducta agresiva o disocial) y con un síndrome neutro como los problemas de atención. Además, la correlación entre el uso de internet y móvil fue fuerte $(\rho=.756 ; p<.001)$. La correlación entre el uso de móvil e internet con competencias psicosociales no resultó significativa.

Tabla 4

Correlaciones entre uso de internet y móvil con problemas YSR

\begin{tabular}{|c|c|c|c|c|c|c|c|c|c|c|c|c|}
\hline & 1 & M & $A D$ & $\mathrm{RD}$ & QS & PS & PP & PA & $C D$ & CA & $\mathrm{SI}$ & SE \\
\hline I & 1 & & & & & & & & & & & \\
\hline M & $.756^{\star \star}$ & 1 & & & & & & & & & & \\
\hline$A D$ & $.255^{* \star}$ & $.303^{\star *}$ & 1 & & & & & & & & & \\
\hline $\mathrm{RD}$ & $.240^{* *}$ & $.184^{*}$ & $.600^{* *}$ & 1 & & & & & & & & \\
\hline QS & $.190^{*}$ & $.185^{*}$ & $.611^{* *}$ & $.504^{* *}$ & 1 & & & & & & & \\
\hline PS & $.272^{\star *}$ & $.239^{* *}$ & $.619^{* *}$ & $.500^{* *}$ & $.499^{* *}$ & 1 & & & & & & \\
\hline PP & $.260^{* *}$ & $.294^{* *}$ & $.528^{* *}$ & $435^{* *}$ & $.476^{* *}$ & $.499^{* *}$ & 1 & & & & & \\
\hline PA & $.360^{\star *}$ & $.364^{\star *}$ & $.347^{* *}$ & $.367^{* *}$ & $.386^{* *}$ & $.381^{* *}$ & $.492^{2 *}$ & 1 & & & & \\
\hline$C D$ & $.374^{* *}$ & $.372^{\star \star}$ & $.202^{\star}$ & $.218^{* *}$ & $.299^{* *}$ & $.264^{* *}$ & $.428^{* *}$ & $.525^{* *}$ & 1 & & & \\
\hline $\mathrm{CA}$ & $.354^{* *}$ & $.368^{* *}$ & $.471^{* *}$ & $.363^{* *}$ & $.446^{* *}$ & $.454^{* *}$ & $.543^{* *}$ & $.579^{* *}$ & $.619^{* *}$ & 1 & & \\
\hline SI & $.272^{\star *}$ & $.271^{\star *}$ & $.895^{* *}$ & $.794^{* *}$ & $.819^{\star *}$ & $.636^{* *}$ & .560 & $.455^{\star *}$ & $.264^{* *}$ & $.500^{\star \star}$ & 1 & \\
\hline SE & $.413^{* *}$ & $.423^{* \star}$ & $.406^{* *}$ & $.342^{* *}$ & $.430^{* *}$ & $.419^{* *}$ & $.551^{* *}$ & $.616^{* *}$ & $.854^{* *}$ & $.928^{\star *}$ & $.455^{\star \star}$ & 1 \\
\hline TP & $.370^{\star *}$ & $.374^{\star \star}$ & $.761^{* *}$ & $.617^{\star *}$ & $.696^{* *}$ & $.679^{* *}$ & $.733^{* *}$ & $.699^{* *}$ & $.580^{\star *}$ & $.780^{\star *}$ & $.822^{\star *}$ & $.778^{\star *}$ \\
\hline
\end{tabular}

${ }^{* *} p<.001 ;{ }^{*} p<.005 ;$ I: Internet; M: Móvil; AD: Ansiedad/Depresión; RD: Retraimiento/Depresión; QS Quejas somáticas; PS: Problemas Sociales; PP: Problemas Pensamiento; PA: Problemas Atención; CD: Conducta Disocial; CA: Conducta Agresiva; SI: Síndromes Internalizantes; SE: Síndromes Externalizantes; TP: Total Problemas YSR. 
Cuando se comparan las puntuaciones de uso de internet y móvil en base a los grupos de las variables que hacen referencia a las competencias psicosociales, como el número de amigos cercanos que tienen, el número de salidas que con ellos realizan, rendimiento escolar en asignaturas de letras y la percepción que ellos tienen de llevarse con sus pares o hermanos en comparación con el resto (tabla 5), no aparecen diferencias significativas entre ellas.

Tabla 5

Competencias psicosociales para las que no hay significación estadística

\begin{tabular}{lcccc}
\hline Variable & Escala & Grados libertad & $\mathrm{F}$ & $\mathrm{p}$ \\
\hline \multirow{2}{*}{ Número de amigos } & Internet & 3,275 & .89 & .794 \\
& Móvil & 3,271 & .88 & .332 \\
\hline \multirow{2}{*}{ Número de salidas con amigos } & Internet & 2,268 & .52 & .403 \\
& Móvil & 2,264 & .28 & .716 \\
\multirow{2}{*}{ Relación con otros jóvenes } & Internet & 2,270 & 1,31 & .915 \\
& Móvil & 2,266 & 1,18 & .607 \\
\multirow{2}{*}{ Relación con los hermanos } & Internet & 2,266 & .24 & .927 \\
& Móvil & 2,262 & .65 & .638 \\
\multirow{2}{*}{ Rendimiento en Letras } & Internet & 3,265 & 1.98 & .077 \\
& Móvil & 3,261 & 1.99 & .081 \\
\hline
\end{tabular}

Sí aparecen diferencias significativas en competencias psicosociales como número de clubs a los que pertenecen, qué relación tienen con sus padres en comparación con sus compañeros, la percepción que tienen de ser autónomos o el rendimiento escolar en matemáticas (tabla 6). Las pruebas post hoc muestran que las puntuaciones superiores en nuevas tecnologías las tienen aquellos que se llevan peor con sus padres ( $M=25,3$ para internet y $M=23,3$ para móvil), en comparación con los que se llevan igual que los demás ( $M=18.15$ para internet y $M=15.75$ para móvil) o mejor que los demás ( $M=18.27$ para internet y $M=16.37$ para móvil); pasan más tiempo con las nuevas tecnologías los que sienten menor autoeficacia que los demás $(M=23,59$ para internet y $M=20.19$ para móvil) que los que sienten la misma autoeficacia que los demás $(M=17.70$ para internet y $M=15.66$ para móvil) o más autoeficacia que los demás ( $M=18,58$ para internet y $M=16,61$ para móvil); quienes no participan en ningún club, organización o asociación, pasan más tiempo con las nuevas tecnologías $(M=$ 19,20 para internet y $M=17,07$ para móvil) que quienes al menos participan en un club, organización o asociación $(M=16,89$ para internet y $M=14,70$ para móvil), dos clubs $(M=17.37$ para internet y $M=$ 15.62 para móvil) o tres clubs ( $M=17.33$ para internet y $M=15.33$ para móvil); y quienes generalmente suspenden en asignaturas de matemáticas, pasan más tiempo con el móvil ( $M=22.46$ para internet y $M=20.29$ para móvil $)$ que quienes están por debajo del promedio $(M=19.14$ para internet y $M=16.77$ para móvil), aprueban en el promedio ( $M=18.27$ para internet y $M=16.04$ para móvil) o aprueban por encima del promedio ( $M=17.27$ para internet y $M=15.46$ para móvil).

Tabla 6

Competencias psicosociales con significación estadística

\begin{tabular}{|c|c|c|c|c|c|}
\hline Variable & Escala & Grados libertad & F o Welch & $p$ & Post-hoc \\
\hline \multirow{2}{*}{ Número de Clubs adscritos } & Internet & 3,272 & 2,53 & $<.001$ & \multirow{2}{*}{$0>(1=2=3)$} \\
\hline & Móvil & 3,268 & 3,55 & $<.001$ & \\
\hline \multirow{2}{*}{ Relación con los padres* } & Internet & 2,271 & 4,99 & $<.001$ & \multirow{2}{*}{$1>(2=3)$} \\
\hline & Móvil & 2,267 & 6,01 & $<.001$ & \\
\hline \multirow{2}{*}{ Hacer cosas por sí mismo* } & Internet & 2,270 & 9,68 & $<.001$ & \multirow{2}{*}{$1>(2=3)$} \\
\hline & Móvil & 2,266 & 5,58 & $<.001$ & \\
\hline \multirow{2}{*}{ Rendimiento en matemáticas } & Internet & 3,267 & 6.47 & $<.001$ & \multirow{2}{*}{$0>(1=2=3)$} \\
\hline & Móvil & 3,263 & 6.66 & $<.001$ & \\
\hline
\end{tabular}

*Se ha utilizado Welch en vez de ANOVA y Gameshowell en vez de HDS Tukey (en pruebas post hoc) por no haber homocedasticidad.

\section{Discusión}

Tras analizar los resultados de este estudio, se observa que con la edad aumenta el abuso del móvil e internet y el abuso de las nuevas tecnologías está relacionado especialmente con problemas de atención y sintomatología externalizante. Mientras que pertenecer al menos a una organización, relacionarse adecuadamente con los padres, ser un individuo autónomo y rendir aceptablemente en asignaturas científicas son variables que protegen frente al abuso del móvil e internet.

Más pormenorizadamente, los resultados muestran con la edad un aumento del uso abusivo del móvil e internet, tal como se encontró en Wu et al. (2019), así como un incremento en problemas 
psicopatológicos internalizantes y externalizantes asociados. Particularmente, los chicos más jóvenes presentan mayores prevalencias de problemática ocasional y frecuente relacionada con el uso del móvil e internet, sin embargo, al llegar al segundo rango de edad estudiada, son las chicas las que muestran más uso problemático que los chicos. Además, se observa con la edad un aumento en psicopatología general, especialmente en las chicas. Estos resultados difieren de lo encontrado por otros autores (Estévez et al 2009; Muñoz-Rivas et al., 2003; Viñas et al., 2002 y Rial et al., 2014), quienes aprecian que el género no está relacionado con el uso de internet y generalmente son los chicos quienes utilizan más las nuevas tecnologías. Estas diferencias pueden ser debidas a que en este estudio el cálculo de las prevalencias se separó por rango de edades, por lo que se aportan datos novedosos del uso de las nuevas tecnologías en adolescentes, según la edad.

Se han encontrado resultados similares a los estudios de Cerniglia et al., 2019; Estévez et al., 2009; Fumero et al., 2020; Jenaro et al., 2007, quienes hallaron que el abuso de las nuevas tecnologías puede influir en situaciones sociales y un escaso autocontrol se relaciona con un mayor uso de las tecnologías (Oliva et al, 2012). En este estudio, la relación existente entre problemas psicopatológicos y el uso abusivo de tecnologías se observa que es entre baja y moderada. Siendo baja la relación para los problemas internalizantes (como Ansiedad/Depresión, Retraimiento/Depresión o Quejas Somáticas) y moderada para los problemas de atención y los problemas externalizantes (Conducta Disocial y Conducta Agresiva). Estos resultados reflejan que el uso abusivo de internet y móvil, además de relacionarse con problemas de atención y autocontrol en los menores, también se relaciona con comportamientos externalizantes que generan cierto malestar social en su comunidad.

Se han analizado variables protectoras en el abuso del móvil e internet y se han detectado como protectoras variables como la pertenencia a organizaciones, autonomía personal, la relación con los padres y el rendimiento en matemáticas. El pertenecer al menos a una organización en la que los menores participan, tener autonomía personal y desenvolverse bien en el mundo, una adecuada relación con los padres y un buen rendimiento en matemáticas, son claves para disminuir el abuso de estas nuevas tecnologías. Otros estudios han identificado también como protector del abuso de internet y móvil la inteligencia emocional (Arrivillaga et al., 2020)

También se ha encontrado que ciertas variables que se esperaba que resultasen protectoras del uso abusivo del móvil e internet no han sido significativas. Variables tales como el número de amigos que tienen, número de salidas que con ellos hacen, la relación que tienen con sus pares y la relación que tienen con sus hermanos no influyen en el abuso de estas tecnologías. Los resultados encontrados por los autores Chóliz y Villanueva, (2011); Cuesta y Gaspar, (2014) podrían explicar por qué no se encuentran en este estudio diferencias significativas en ciertas competencias psicosociales, ya que el uso del móvil e internet es una fuente de ocio y se relaciona con una mejor socialización que permite una subjetiva sensación de proximidad.

Estos datos amplían la ya numerosa investigación en el campo de la adicción a las nuevas tecnologías, siendo el estudio más amplio hasta la fecha en la población de adolescentes de la Región de Murcia, en concreto en la comarca del Mar Menor. También se han actualizado los puntos de corte de dos escalas que miden el uso problemático de las nuevas tecnologías y se han establecido relaciones con problemas psicopatológicos. De los resultados de este estudio se pone de evidencia la importancia de crear planes de prevención sobre el uso inadecuado de las nuevas tecnologías, donde es necesario enseñar a los colectivos de riesgo el correcto uso de las mismas.

En cuanto a las limitaciones del estudio, se pone de relieve que la principal limitación fue el muestreo incidental, lo que no asegura la representatividad de la muestra y que la recogida de muestra se centró únicamente en la comarca del Mar Menor.

Para futuras investigaciones, sería conveniente valorar si la medición de Competencias Psicosociales, que han aparecido como protectoras frente al abuso del móvil e internet en adolescentes, necesitan de otro instrumento más específico para ser evaluadas.

Finalmente se concluye que tal vez ciertas actividades extraescolares, una relación positiva con los padres, donde se fijen unos límites claros que favorezcan la autonomía del adolescente y una adecuada "dieta digital" sean una buena solución para prevenir el abuso del móvil e internet y los problemas psicopatológicos asociados.

\section{Referencias}

1. Achenbach, T.M. y Edelbrock, C.S. (1987). Manual for the Youth Self Report and profile. Burlington VT: University of Vermont.

2. Andreassen, C.S., Billieux, J., Griffiths, M.D., Kuss, D.J., Demetrovics, Z., Mazzoni, E., Pallesen, S. (2017). Adicción a internet, redes sociales y videojuegos. Revista de Toxicomanías, 81. 
3. Arrivillaga, C., Rey, L., y Extremera, N. (2020). Adolescents problematic internet and Smartphone use is related to suicide ideation: Does emotional intelligence make a difference? Computers in human behavior, 110, 106375. https://doi.org/10.1016/i.chb.2020.106375

4. Beranuy, M., Chamarro, A., Graner, C. y Carbonell, X. (2009). Validación de dos escalas breves para evaluar la adicción a Internet y el abuso de móvil. Psicothema, 21, 480-485.

5. Beranuy, M., Oberst, U., Carbonell, X. y Chamarro, A. (2009). Problematic Internet and mobile phone use and clinical symptoms in college students: The role of emotional intelligence. Computers in Human Behaviour 25, 1182-1187. https://doi.org/10.1016/j.chb.2009.03.001

6. Carbonell, X., Guardiola, E., Beranuy, M., y Bellés, A. (2009). A bibliometric analysis of the scientific literature on Internet, video games, and cell phone addiction. Journal of the Medical Library Association: JMLA, 97, 102-107. https://doi.org/10.3163/1536-5050.97.2.006

7. Castellana, M., Sánchez-Carbonell, X., Graner C., y Beranuy, M. (2007). El adolescente ante las tecnologías de la información y la comunicación: Internet, móvil y videojuegos. Papeles del psicólogo, 28, 196-204.

8. Cerniglia, L., Guicciardi, M., Sinatra, M., Monacis, L., Simonelli, A., y Cimino, S. (2019). The use of digital technologies, impulsivity and psychopathological symptoms in adolescence.Behavioral Sciences, 9, 82. https://doi.org/10.3390/bs9080082

9. Chóliz, M. Villanueva, V. y Chóliz, M.C. (2009). Ellos, ellas y su móvil: Uso, abuso (¿y dependencia?) del teléfono móvil en la adolescencia. Revista Española de Drogodependencias, 34, 74-88.

10. Chóliz, M., y Villanueva, V. (2011). Evaluación de la adicción al móvil en la adolescencia. Revista española de drogodependencias, 2, 165-183.

11. Cuesta, U., y Gaspar, S. (2014). Análisis motivacional del uso del smartphone entre jóvenes: una investigación cualitativa. Historia y Comunicación Social, 18. https://doi.org/10.5209/rev HICS.2013.v18.44252

12. Echeburúa, E., Labrador, F. J., y Becoña, E. (2009). Adicción a las nuevas tecnologías. Madrid: Pirámide.

13. Espinar, E., y López, C. (2009). Jóvenes y adolescentes ante las nuevas tecnologías: percepción de riesgos. Athenea digital: revista de pensamiento e investigación social, 16, 001-020.

14. Estévez, L., Bayón, C., de la Cruz, J. y Fernández-Líria, A. (2009). Uso y abuso de Internet en adolescentes. En E. Echeburúa, F.J. Labrador y E. Becoña (eds.). Adicción a las nuevas tecnologías (pp. 101-130). Madrid: Pirámide.

15. Fumero, A., Marrero, R. J., Bethencourt, J. M., y Peñate, W. (2020). Risk factors of internet gaming disorder symptoms in Spanish adolescents. Computers in human behavior, 111, 106416. https://doi.org/10.1016/j.chb.2020.106416

16. Gil, Adriana; Feliu, Joel; Rivero, Isabel; Gil, y Eva P. (2003). ¿Nuevas tecnologías de la información o nuevas tecnologías de relación? Niños, jóvenes y cultura digital. Artículo en línea. Universitat Oberta de Catalunya. Extraído el 16 de octubre de 2019 de https://www.uoc.edu/dt/20347/index. $\underline{\mathrm{html}}$

17. Gómez, P., Rial, A., Braña, T., Varela, J. y Barreiro, C. (2014). Evaluation and early detection of problematic Internet use in adolescents. Psicothema, 26, 21-26. https://doi.org/10.7334/psicothema2013.109

18. Gracia, M., Vigo, M., Fernández, M.J., M, M. (2002). Problemas conductuales relacionados con el uso de internet: Un estudio exploratorio. Anales de psicología, 18, 273-292.

19. Instituto de Adicciones de Madrid (2008) Estudio de Uso Problemático de las tecnologías de la información, la comunicación y el juego entre los adolescentes y jóvenes de la ciudad de Madrid. Madrid: Doblehache Comunicación

20. Instituto Nacional de Estadística. (2005). Instituto Nacional de Estadística. Madrid: INE. Recuperado el 21 de octubre de 2019 de http://www.ine.es/

21. Jenaro, C., Flores, N., Gómez-Vela, M., González-Gil, F. y Caballo, C. (2007). Problematic Internet and cellphone use: Psychological, behavioral, and health correlates. Addiction Research and Theory, 15, 309-320. https://doi.org/10.1080/16066350701350247

22. Labrador F. J. y Villadangos, S. M ${ }^{\mathrm{a}}$. (2009). Adicciones a nuevas tecnologías en adolescentes y jóvenes. En E. Echeburúa, F.J. Labrador y E. Becoña (eds.). Adicción a las nuevas tecnologías (44-75). Madrid: Pirámide.

23. Labrador, F.J. y Villadangos, S.M. (2010). Menores y nuevas tecnologías: conductas indicadoras de posible problema de adicción. Psicothema, 22, 180-188.

24. Lemos, S., Fidalgo, A. M., Calvo, P., y Menéndez, P. (1992). Salud mental de los adolescentes asturianos. Psicothema, 4, 21-48. 
25. López-Fernández, O., Honrubia-Serrano, Ma . L. y Freixa-Blanxart (2012). Adaptación española del "Mobile Phone Problem Use Scale". Adicciones, 24, 123-130. https://doi.org/10.20882/adicciones.104

26. López-Soler, C. y Romero, A (2013). TDAH y trastorno del comportamiento en la infancia y la adolescencia. Clínica, diagnóstico, evaluación y tratamiento. Madrid: Pirámide.

27. Muñoz-Rivas, M. J., Navarro, M. E. y Ortega, N. (2003). Patrones de uso de Internet en población universitaria española. Adicciones, 15, 137-144. https://doi.org/10.20882/adicciones.437

28. Oliva, A., Hidalgo, Ma . V., Moreno, C., Jiménez, L., Jiménez, A., Antolín, L. y Ramos, P. (2012). Uso y riesgo de adicciones a las nuevas tecnologías entre adolescentes y jóvenes andaluces. Sevilla: Editorial Agua Clara S.L.

29. Rial, A., Gómez, P., Braña, T. y Varela, J. (2014). Actitudes, percepciones y uso de Internet y las redes sociales entre los adolescentes de la comunidad gallega (España). Anales de Psicología, 30, 642-655. http://dx.doi.org/10.6018/analesps.30.2.159111

30. Roca, G. (Coord.) (2015) Las nuevas tecnologías en niños y adolescentes. Guía para educar saludablemente en una sociedad digital. Barcelona: Hospital Sant Joan de Déu (ed).

31. Sánchez-Martínez, M y Otero, A. (2009). Factors associated with cell phone use in adolescents in the community of Madrid (Spain). Cyberpsychology and Behavior, 12, 131-137. https://doi. org/10.1089/cpb.2008.0164

32. Viñas, F., Juan, J., Villar, E., Caparros, B., Perez, I. y Cornella, M. (2002). Internet y psicopatología: las nuevas formas de comunicación y su relación con diferentes índices de psicopatología. Clínica y Salud, 13, 235-256.

33. Wu, C. Y., Lee, M. B., Liao, S. C., y Ko, C. H. (2019). A nationwide survey of the prevalence and psychosocial correlates of internet addictive disorders in Taiwan. Journal of the Formosan Medical Association, 118, 514-523. https://doi.org/10.1016/j.jfma.2018.10.022

34. Young, K. (2004). Internet Addiction: A New Clinical Phenomenon and Its Consequences. American Behavioral Scientis, 48, 402-415. https://doi.org/10.1177/0002764204270278

RECIBIDO: 25 de noviembre de 2019

MODIFICADO: 30 de junio de 2020

ACEPTADO: 16 de julio de 2020 